\title{
Directional genomic hybridization for chromosomal inversion discovery and detection
}

\author{
F. Andrew Ray • Erin Zimmerman • \\ Bruce Robinson - Michael N. Cornforth • \\ Joel S. Bedford • Edwin H. Goodwin • \\ Susan M. Bailey
}

Received: 17 January 2013 / Revised: 28 February 2013 / Accepted: 12 March 2013 /Published online: 10 April 2013

(C) The Author(s) 2013. This article is published with open access at Springerlink.com

\begin{abstract}
Chromosomal rearrangements are a source of structural variation within the genome that figure prominently in human disease, where the importance of translocations and deletions is well recognized. In principle, inversions - reversals in the orientation of DNA sequences within a chromosome- should have similar detrimental potential. However, the study of inversions has been hampered by traditional approaches used for their detection, which are not particularly robust. Even with significant advances in whole genome approaches, changes in the absolute orientation of DNA remain difficult to detect routinely. Consequently, our understanding of
\end{abstract}

Responsible Editor: Wendy Bickmore.

Electronic supplementary material The online version of this article (doi:10.1007/s10577-013-9345-0) contains

supplementary material, which is available to authorized users.

F. A. Ray · J. S. Bedford · S. M. Bailey

Department of Environmental \& Radiological Health

Sciences, Colorado State University, Fort Collins, CO

80523-1618, USA

M. N. Cornforth

Department of Radiation Oncology,

University of Texas Medical Branch,

Galveston, TX 77555-0884, USA

F. A. Ray · E. Zimmerman • B. Robinson •

M. N. Cornforth · J. S. Bedford • E. H. Goodwin •

S. M. Bailey $(\bowtie)$

KromaTiD Inc, 320 East Vine Dr., Suite 316,

Fort Collins, CO 80524-2329, USA

e-mail: Susan.Bailey@colostate.edu inversions is still surprisingly limited, as is our appreciation for their frequency and involvement in human disease. Here, we introduce the directional genomic hybridization methodology of chromatid painting - a whole new way of looking at structural features of the genome - that can be employed with high resolution on a cell-by-cell basis, and demonstrate its basic capabilities for genome-wide discovery and targeted detection of inversions. Bioinformatics enabled development of sequence- and strand-specific directional probe sets, which when coupled with singlestranded hybridization, greatly improved the resolution and ease of inversion detection. We highlight examples of the far-ranging applicability of this cytogenomics-based approach, which include confirmation of the alignment of the human genome database and evidence that individuals themselves share similar sequence directionality, as well as use in comparative and evolutionary studies for any species whose genome has been sequenced. In addition to applications related to basic mechanistic studies, the information obtainable with strand-specific hybridization strategies may ultimately enable novel gene discovery, thereby benefitting the diagnosis and treatment of a variety of human disease states and disorders including cancer, autism, and idiopathic infertility.

Keywords chromatid painting · chromosomal inversions $\cdot$ Strand-specific hybridization
Abbreviations
a-CGH Microarray-based comparative genomic hybridization 
BrdU 5'-Bromo-2'-deoxyuridine

CNVs Copy number variants

CO-FISH Chromosome orientation

fluorescence in situ hybridization

FISH Fluorescence in situ hybridization

\section{Introduction}

Abnormalities associated with chromosome rearrangements have been studied for many years, and an appreciation for their importance emerged in parallel with development of methods that enabled their detection. More than 800 tumor-specific chromosome aberrations, primarily translocations and large deletions, have been identified (Mitelman 2000). Detailed analysis of the breakpoints involved in these structural changes has been instrumental in the discovery of many cancerrelated genes and, more broadly, has shed light on underlying mechanisms of carcinogenesis. Particularly illustrative early examples include identification of the 9:22 translocation associated with chronic myeloid leukemia (Nowell and Hungerford 1960; Rowley 1973), and the 8:14 translocation that results in Burkitt's lymphoma (Haluska et al. 1986). These and other notable success stories would not have been possible without corresponding advances in cytogenetic methodologies (Rowley 1990).

Contemporary approaches such as fluorescence in situ hybridization (FISH) and microarray-based a-CGH have expanded our view and appreciation of alterations associated with chromosome aberrations. For example, many apparently balanced translocations are actually complex rearrangements that concurrently involve deletions and duplications (Chen et al. 2010; Manning and Hudgins 2010), as well as inversions (Gribble et al. 2005). Chromosomal inversions are intra-chromosomal rearrangements that result from two breaks occurring within the same chromosome, followed by re-insertion of the broken segment in the opposite (inverted) orientation, and are among the most difficult of structural rearrangements to detect.

Traditionally inversions, and other types of structural rearrangements, have been detected as disruptions to the visual patterns produced by various types of bandingmegabase-sized striations distributed laterally along the lengths of chromosomes (Bickmore and Craig 1977) an approach that severely limits the size of inversion that can be detected. Moreover, because such pattern disruptions do not necessarily visibly alter banding patterns (Savage 1977a, b), and because they are undetectable to whole chromosome painting by FISH, even large inversions can be difficult to detect with regularity (Savage 1977a, b). While it is undeniable that a significant subset of inversions is visible by traditional cytogenetic approaches, it is also the case that many (perhaps even most) remain undetected.

Many of the problems relating to pattern recognition and resolution that plague banding analysis can, in principle, be overcome through more molecular approaches. A case in point is the recent discovery of a (p13.3q24.3) pericentric inversion encompassing practically all of chromosome16 that was identified by transcriptome sequencing, but which otherwise was "cryptic" to alternative methods of analysis (Gruber et al. 2012). The discovery, which involved the concerted effort of several institutions and multiple authors, was important for the additional reason that the inversion encoded a fusion protein (CBFA2T3-GLIS2) that defined a particularly aggressive subtype of pediatric acute leukemia.

It is fair to say that molecular methods face their fair share of challenges regarding inversion detection as well, and that despite significant progress made toward characterizing structural variation using such approaches, our understanding of inversions is still surprisingly limited (Baker 2012). Whereas CNVs can be mapped with great precision, changes in orientation (i.e., phase) of DNA sequences are much more difficult to detect. Techniques like paired-end sequencing have great potential to identify and map inversions across the human genome (Tuzun et al. 2005), but can be limited by possible mis-assembly of reference genomes and the presence of flanking high-density inverted duplications (Feuk 2010).

These issues aside, alternative independent methodologies would be of value for validating and characterizing recurrent structural variants identified by sequencing approaches - as, for example, those recently reported for colorectal cancer (Cancer Genome Atlas 2012) and adenocarcinoma of the lung (Imielinske 2012). Highlighting the potential significance of inversions in the etiology of disease, and at the same time underscoring difficulties in detecting and characterizing them (Antonacci et al. 2009), a majority of variants identified in this latter study (Imielinske 2012) were relegated to the generic classification of "intra- 
chromosomal rearrangements." One might well suspect that many of the variants in this class were bona fide inversions.

Lastly, it should be noted that sequencing approaches are not particularly well suited to addressing the cellular heterogeneity typical of solid tumors since this would ostensibly involve characterization of abnormalities among mosaic sub-populations. This would also apply to single cell sequencing methodologies (Falconer et al. 2012), which are impractical for use on more than a few cells. For similar reasons, strictly genomics-based approaches would be of dubious value in constructing quantitatively meaningful cellular dose responses-as used for the purpose of risk assessment following exposure to genotoxic agents-because this requires the collective assembly of data from of many cells, each individually assessed for damage.

There exists, therefore, a need for methodologies of chromosomal inversion detection that provide the higher resolution of molecular approaches, while still retaining one of cytogenetics' most powerful assets - its ability to provide an unbiased view of the entire genome on an individual cell basis. Here, we introduce the directional genomic hybridization methodology of chromatid painting — not to be confused with the standard practice of chromosome painting - that promises to circumvent many of the aforementioned limitations associated with inversion detection. Chromatid painting evolved from the strand-specific hybridization concept of chromosome orientation FISH (CO-FISH; Bailey et al. 2004a, b, c; Goodwin and Meyne 1993; see Fig. 1). Due to the requirement for single-stranded probes however, the methodology was limited to the interrogation of repetitive centromeric or telomeric sequences with simple oligonucleotide probes, where it was used to study a variety of cytogenetic phenomena including: lateral asymmetry (Goodwin et al. 1996); leading- versus lagging-strand telomeres (Bailey et al. 2001); telomere-DNA double strand break fusions (Bailey et al. 2004a, b, c); recombination within sub-telomeric and telomeric regions (T-SCE; Bailey et al. 2004a, b, c;

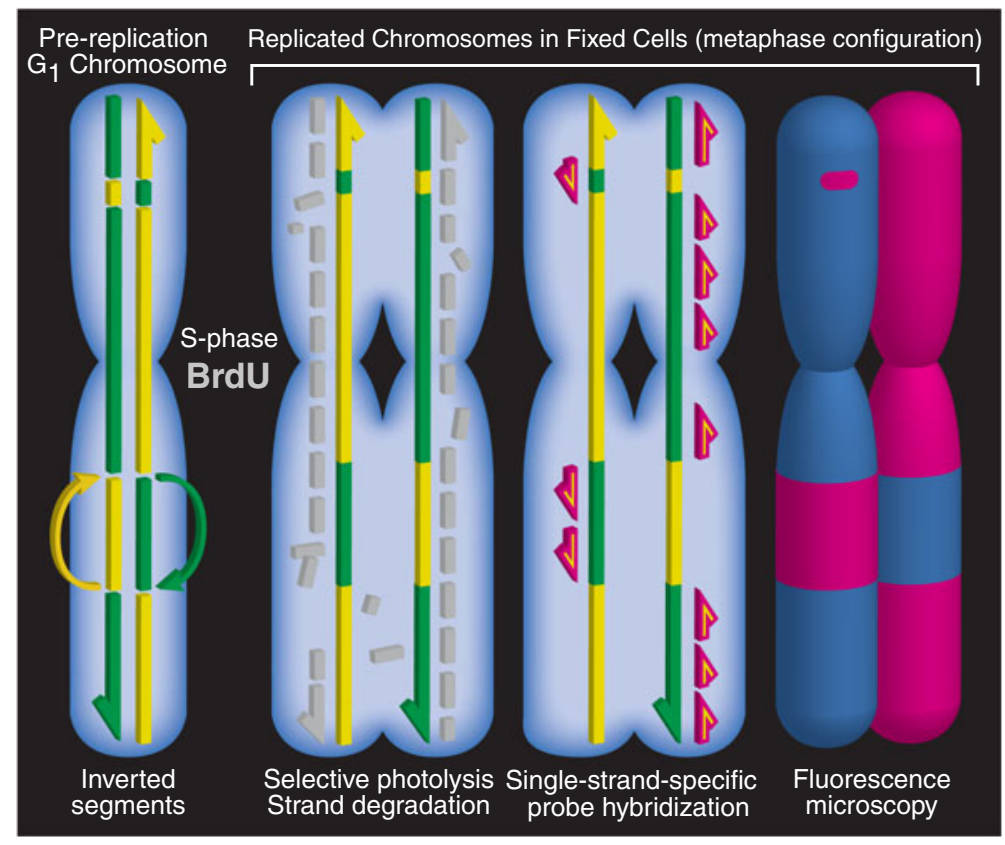

Fig. 1 Chromatid painting and inversion detection. Following S-phase, each complementary polynucleotide strand from a $G_{1}$ chromosome segregates into respective sister chromatids at metaphase. When BrdU is incorporated during DNA synthesis, each nascent strand becomes photo-labile, allowing it to be selectively degraded. For the purposes of in situ hybridization, this results in a metaphase chromosome whose sister chromatids are single-stranded and complementary. Because inverted DNA sequences must reverse their $5^{\prime} \rightarrow 3^{\prime}$ orientation in order to preserve polarity, individual strands within the inversion are obligated to "switch places" between the sister chromatids; therefore, hybridization of fluorescently tagged directional single-stranded probes to unique sequences along either chromatid results in a microscopically visible signal that "switches" from one chromatid to its sister. For large inversions, the signal switch is accompanied by a corresponding lack of signal on the opposite chromatid; for small inversions, the unlabeled segment is often obscured by the brightness of the fluorescent signal 
Cornforth and Eberle 2001); and mammalian telomere replication timing (Zou et al. 2004).

With availability and refinement of the human genome database came the ability to utilize bioinformatics-based design schemes for construction of single-stranded oligonucleotide probes to unique sequences along the length of a given chromosome that were also similar with respect to their absolute $5^{\prime}$ pter $\rightarrow 3$ 'qter directionality (orientation), an advancement that greatly expanded the potential of strandspecific hybridization strategies. For example, by pooling together of many such unique sequence, strand-specific probes it became possible to effectively "paint" individual whole chromatids of a given chromosome, or any specific region thereof. In so doing, changes in directionality (inversions) occurring anywhere along the length of a painted chromosome readily reveal themselves as an abrupt "switch" in the location of hybridization signalfrom one sister chromatid to the other. This cytogenomics-based approach of achieving strandspecificity provides investigators a new tool for inversion discovery and high-resolution inversion detection.

\section{Materials and methods}

\section{Cell culture}

Human cells (normal fibroblasts and lymphocytes; Kasumi-4 leukemia cell line; HTori-3 immortalized thyroid cells), and chimpanzee, gorilla, and orangutan fibroblasts, were cultured for a single cell cycle in complete media containing 5.0 $\mu \mathrm{M}$ 5-bromo-2-deoxyuridine and $1.0 \mu \mathrm{M}$ 5-bromo-deoxycytidine (BrdU/BrdC; Acros, Chem Impex International). Cells were blocked in mitosis for 2-4 h using Colcemid (KaryoMax, Gibco) at a final concentration of $0.1 \mu \mathrm{g} / \mathrm{ml}$. Mitotic cells were harvested and dropped onto slides using standard cytogenetic protocols (Bailey et al. 2010).

Directional strand-specific probes

Creation of the human chromosome 3 specific chromatid paint began by downloading contiguous DNA sequences from the publically available NCBI genomic database (GRCh37.p2 primary assembly) and masking them using Genetic Information Research Institute software; i.e., repeat sequences were removed. Single-stranded oligonucleotide probes (oligos) were designed to unique sequences and our specifications using ARRAY Designer (version 4.2), and later with proprietary software (KromaTiD Inc.), tiling to small target regions at specified non-overlapping locations along the length of each chromosome 3 contig at $\sim 1-\mathrm{Mb}$ intervals. Oligo design criterion demanded similar directionality and length ( 40-mers), as well as uniform melting temperatures $\left(70.0 \pm 5.0^{\circ} \mathrm{C}\right)$. More than 17,000 individual oligos were synthesized (Invitrogen), hydrated, and pooled in subsets of 45 for end-labeling with fluorescent dNTP analogs (Cy-3, Fluorescein; General Electric, Perkin Elmer) using terminal transferase (New England Biolabs). Pools of 90 individual labeled oligos constituted a probe set, which together spanned relatively short unique regions $(\sim 5-$ $14.5 \mathrm{~kb}$ ); probe sets were hybridized individually to confirm strand specificity. The complete chromosome 3 -specific chromatid paint consisted of 190 probe sets.

In similar fashion, targeted probe sets to both sides of known inversion breakpoints on chromosome 3 ( $q 21$; $q 26)$ and chromosome $10(q 11.2 ; q 21)$ were also generated. Targeted probe sets were larger in that they consisted of 180-200 individual labeled oligos and they covered larger unique regions $(\sim 14-63 \mathrm{~kb})$, modifications that served to increase brightness and visibility of the individual signals. Proximity of the selected probe sets to the specific inversion breakpoints did not exceed $1 \mathrm{Mb}$.

Single-stranded hybridization pre-treatment

Briefly, slides $(25 \times 75 \times 1 \mathrm{~mm})$ were incubated in PN buffer (sodium phosphate) for $10 \mathrm{~min}$ at room temperature, rinsed in phosphate-buffered saline, then dehydrated through an ethanol series $(75,85$, and $100 \%)$ for 2 min each. Slides were air dried, stained with Hoechst 33258 $(0.5 \mu \mathrm{g} / \mathrm{ml}$ in $2 \times$ sodium citrate; SSC) for $15 \mathrm{~min}$ in the dark, then rinsed with deionized distilled water $\left(\mathrm{ddH}_{2} \mathrm{O}\right)$. Slides were air dried, flooded with $2 \times$ SSC, coverslipped (1 mm), and exposed to $365 \mathrm{~nm}$ ultraviolet light (UV Stratalinker 2400; Strategene) for $35 \mathrm{~min}$, then rinsed in $\mathrm{ddH}_{2} \mathrm{O}$ to remove coverslip, air dried, and dehydrated in the ethanol series, as above (Bailey et al. 2010). For use of single-stranded directional probe sets with standard (double-stranded) FISH, slide pretreatment was not performed prior to hybridization.

Strand-specific hybridization

For each pretreated slide, a mixture of hybridization buffer (25.3 $\mu \mathrm{l}$; KromaTiD), chromatid paint (2.25 $\mu \mathrm{l}$; 
KromaTiD), and $\mathrm{ddH}_{2} \mathrm{O}(2.45 \mu \mathrm{l})$ was prepared and heated to $75{ }^{\circ} \mathrm{C}$ for $5 \mathrm{~min}$, then pipetted onto pretreated slides, which were coverslipped and sealed with rubber cement. Slides were heated at $73{ }^{\circ} \mathrm{C}$ for $3 \mathrm{~min}$, then transferred to individual hybridization chambers (Corning) and incubated at $37^{\circ} \mathrm{C}$ overnight. After hybridization, the slides were washed five times in $2 \times$ SSC at $42{ }^{\circ} \mathrm{C}$ for $15 \mathrm{~min}$ each. Slides were rinsed in PN buffer, counterstained with DAPI/antifade (18 $\mu$ l; Vector Labs), and coverslipped.

Image capture and analysis was performed on an Olympus Bx41 microscope outfitted with fluorochrome-appropriate excitation/barrier filters, running Metavue 7.1 software and equipped with a Photometrics CoolSNAP ES ${ }^{2}$ camera. Efficiency of probe hybridization was routinely greater than $90 \%$. Number of metaphases scored was dependent on endpoint (e.g., recurrent inversions only required 3-5) and treatment (e.g., dose).

\section{Results}

Directional genomic hybridization methodology

A prerequisite for achieving strand-specific hybridization is that cells incorporate bromo-deoxyuridine/ deoxycytidine $(\mathrm{BrdU} / \mathrm{BrdC})$ during a single round of replication, so that sister chromatids are unifilarly substituted (Fig. 1). Prior to hybridization with singlestranded probes, slides are stained with Hoechst 33258, exposed to UV light to nick the DNA at sites of BrdU incorporation, and treated with Exonuclease III to selectively degrade the newly replicated strands (Bailey et al. 2010). For the purposes of subsequent hybridization, this strategy effectively renders the entirety of each sister chromatid a single-stranded target.

Bioinformatics-based approaches were developed and employed to guide the design of sequencespecific, single-stranded oligonucleotides, which were also of similar directionality. We initially reasoned that the orientation of targets along a single contig had the highest probability of being of similar $5^{\prime} \rightarrow 3^{\prime}$ direction; therefore, the largest contig on chromosome $3 \mathrm{q}$ (NT_005612) was selected (Fig. 2a). Probe sets to unique sequences were designed, synthesized, labeled (green), and hybridized to pre-treated human metaphase chromosomes, which produced a strand-specific signal at the predicted location. Consistent contig alignment along the entire length of chromosome 3 was confirmed by designing probe sets to the remaining three contigs, each of which contained 90 individual oligonucleotides that were labeled (red) and hybridized similarly. All produced strand-specific signals at the predicted locations along chromosome 3 , and all were on the same chromatid, thereby validating similar $5^{\prime} \rightarrow 3^{\prime}$ direction of the assembled contigs (Fig. 2b). Extending this cytogenomic strategy, some 17,000 individual (singlestranded) oligonucleotides were synthesized, pooled, and fluorescently labeled to create a complete chromosome 3 chromatid-specific paint. Figure 3 a illustrates the robustness and specificity of the chromatid paint hybridized to a human whole-metaphase spread (effectively single-stranded due to pre-treatment), where hybridization is confined to one, and only one, chromatid of the target chromosome.

Genome-wide inversion discovery

Due to the necessity of maintaining DNA 5'-to-3' polarity, inverted segments reinsert themselves into chromosomal DNA in the reversed (or opposite) orientation. Because the sequence- and strand-specific probes all possess the same $5^{\prime} \rightarrow 3^{\prime}$ directionality (similar orientation) and thus are capable of hybridizing only to complementary stretches of single-stranded chromatids, inversions are revealed as obligatory color switches of signal from one sister chromatid to the other (Fig. 1). Ionizing radiation (IR) is well known for its ability to induce chromosome rearrangements (Cornforth 1998), including inversions (MuhlmannDiaz and Bedford 1995), and was used here to demonstrate the utility of chromatid painting for discovery of novel inversions. Figure 3b, c shows small and large inversions, respectively, following exposure of human cells to IR (gamma rays).

Assuming all probe sets yield visible signals, chromatid painting with probes spaced at $1 \mathrm{Mb}$ intervals will reveal any inversion of $1 \mathrm{Mb}$ length or larger. Inversions less than $1 \mathrm{Mb}$ may also be detected if by chance a probe set falls within the inverted region. This happens with a frequency that depends on inversion size. For example, $50 \%$ of $0.5 \mathrm{Mb}$ inversions occurring at random locations produce visible signals; similarly $10 \%$ of $0.1 \mathrm{Mb}$ inversions are detectable. Detection of much smaller inversions is possible with more densely spaced probes. To illustrate this important point, we designed a mock "mini- 
a

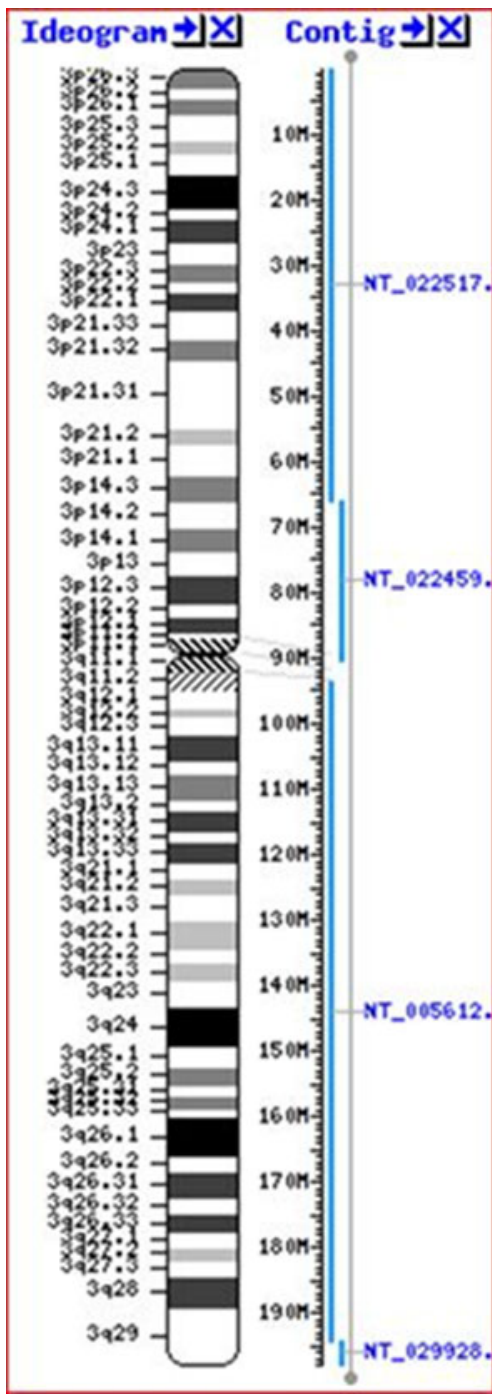

b
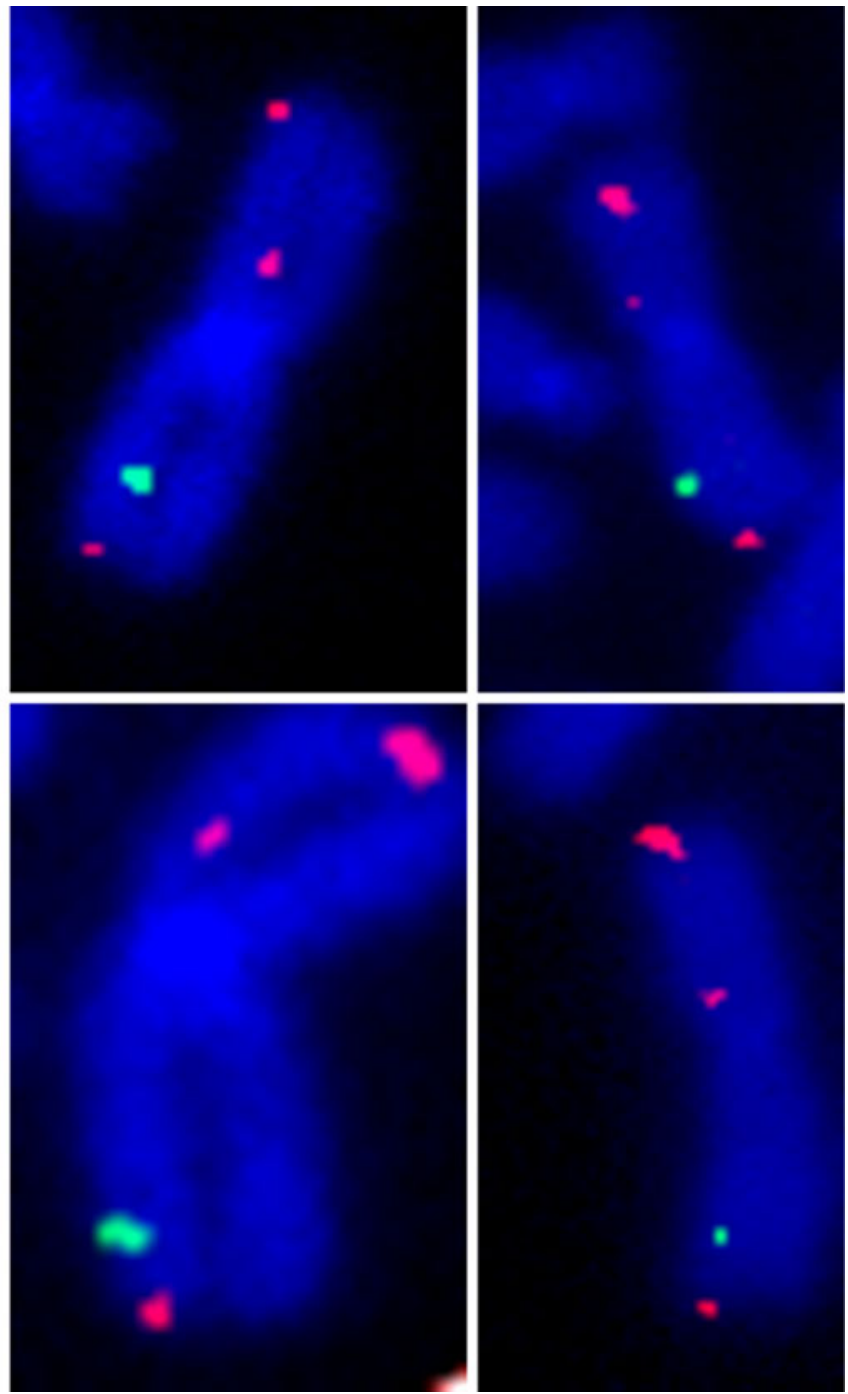

Fig. 2 Confirmation of chromosome 3 contig alignment. a Human genome database contig map: http:/www.ncbi.nlm.nih.gov/ projects $/$ mapview $/$ maps.cgi?taxid $=9606 \& \mathrm{chr}=3$ b Probe sets to all four contigs along chromosome 3 were designed, synthesized,

inversion" within a $10 \mathrm{Mb}$ region of chromosome 3q. Probe sets covering the region were tagged with fluorescein (green); excluded was a $6 \mathrm{~kb}$ segment nested within the region. For this segment, oligos were intentionally designed in the reverse orientation, synthesized and tagged with Cy3 (red). This "simulated" 6 $\mathrm{kb}$ inversion serves as further validation, provides a useful reference marker, and demonstrates that even quite small inversions are cytogenetically detectable utilizing directional genomic hybridization strategies (Fig. 3d). labeled, and hybridized to normal human fibroblasts following strand-specific pre-treatment; one probe set to the large NT 005612 contig (green) and probe sets to the other three contigs (red) are shown

High-resolution targeted inversion detection

The ability to detect and characterize inversions can be further augmented using high-resolution, strand-specific probe sets targeted to previously verified or suspected breakpoints. The Kasumi 4 cell line, derived from a patient with chronic myelogenous leukemia (CML), possesses a variant chromosome 3 homolog with a large $q 21$; $q 26$ inversion (Asou et al. 1996). Strand-specific hybridization of Kasumi 4 metaphases with closely spaced probe sets-targeted across the known region of the 


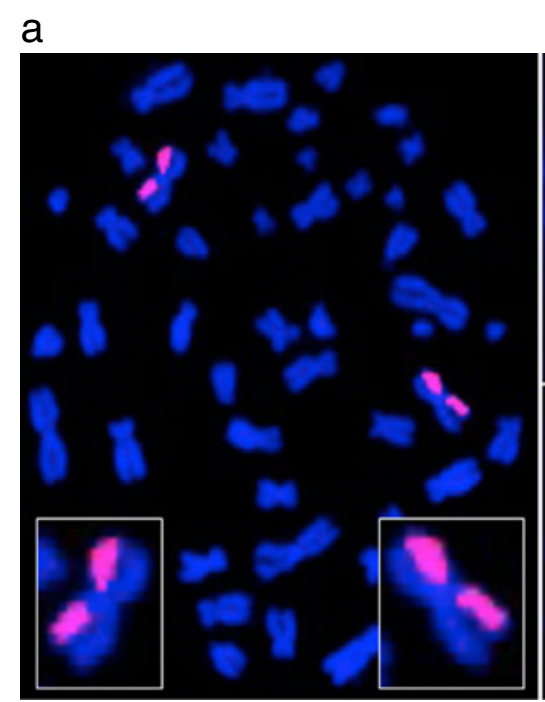

b

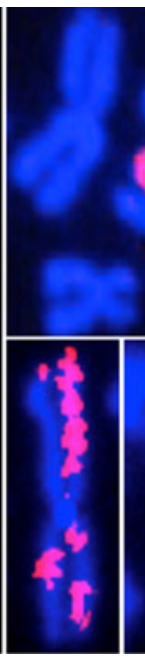

C

Fig. 3 Inversion discovery and detection with directional genomic hybridization. a Directional, fluorescently tagged (red) singlestranded probe sets to unique sequences along one chromatid of human chromosome 3 are both chromosome- and chromatidspecific (insets are higher magnification). b, c Ionizing radiationinduced small and large inversions, respectively. $\mathbf{d}$ A simulated 6-

breakpoints and labeled with different fluorochromesreadily identified the inverted homolog as the one in which the red segment switched to the opposite chromatid (Fig. 3e, arrow). The green signals on its sister chromatid represent probes directed to sequences proximal and distal to the inversion breakpoints. The RET/PTC1 rearrangement associated with radiation-induced papillary thyroid carcinoma provides another noteworthy example as it involves a $q 11.2 ; q 21$ inversion in one homolog of chromosome 10 (Caudill et al. 2005). Here, HTori-3 immortalized human thyroid cell cultures were irradiated and interrogated with strand-specific probe sets targeted to the inverted region on chromosome 10. Consistent with Caudill et al., analysis at time zero revealed a small subset of cells in the exposed population that contained the $\operatorname{inv}(10)(q 11.2 ; q 21)$ rearrangement in one of the homologues, as evidenced by the obvious splitting of the strand-specific signals across sister chromatids (Fig. 3f; arrow).

Other applications of directional genomic hybridization

The human chromatid-3 paint also hybridized to closely related hominoid species, specifically chimpanzee,

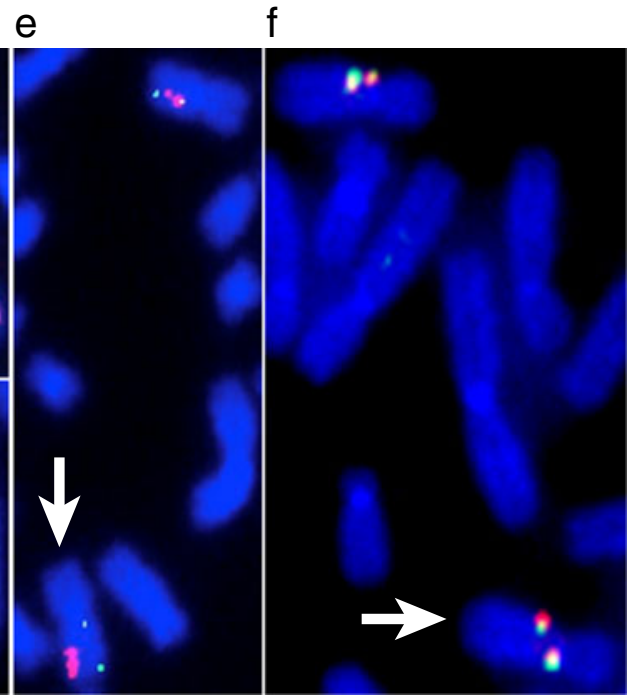

d $\mathrm{kb}$ inversion (red) on one chromatid of a normal unirradiated cell. The region is flanked by complementary probe sets (green), which hybridize to the opposite chromatid. e Targeted high-resolution, two-color detection of known inversion breakpoints associated with $\operatorname{inv}(3)$ in CML, and $\mathbf{f} \operatorname{inv}(10)$ associated with thyroid cancer; arrows depict inverted homologues

gorilla, and orangutan (Supplementary Fig. 1), supportive of its application in comparative and evolutionary studies. Visible gaps in hybridization signal likely represent repetitive regions, for example around the centromeres, or stretches of sequence not identical to human, as these would not be present in the chromatid paint, and therefore are not detected. Consistent with other observations, variation in the state of chromosome compaction also appeared to influence signal intensities. Directional strand-specific hybridization revealed no recurrent inversions in either chimpanzee (Supplementary Fig. 1a) or gorilla (not shown). In contrast, a large inversion was noted in both homologs of orangutan chromosome 3 (Supplementary Fig. 1b).

Chromatid painting facilitates simultaneous detection of both intra-chromosomal changes (inversions) and inter-chromosomal changes (dicentrics, translocations). The damage-induced breakage and mis-repair of two chromosomes that results in dicentric formation also produces a compound fragment consisting of the two broken acentric pieces, which also mis-rejoin. These events are clearly visible with chromatid painting (Supplementary Fig. 2), where one broken chromosome 3 is involved with an unidentified broken chromosome to form a dicentric chromosome; the associated compound acentric 
fragment is definitively identified as it consists of the remaining pieces of chromosome 3 and the other chromosome. Chromatid painting concurrently revealed a large inversion (color switch to sister chromatid) in the uninvolved chromosome 3 (Supplementary Fig. 2b).

The advantages of oligonucleotide probes for standard FISH applications have been recently demonstrated (Boyle et al. 2011; Yamada et al. 2011). Noteworthy of such strategies is the superb specificity of the probes themselves, which do not require unlabeled repetitive DNA (Cot1) to block non-specific binding during hybridization. This valuable feature is true of our strand-specific directional probe sets as well, regardless of whether they are being used for (single-stranded) chromatid painting, or with standard (double-stranded) FISH for conventional chromosome painting (Supplementary Fig. 3).

\section{Discussion}

Utilizing strand-specific hybridization strategies coupled with bioinformatics for sequence- and strandspecific directional probe design, we have developed chromatid (as opposed to chromosome) painting. This cytogenomics-based methodology offers highresolution detection of chromosomal inversions on a cell-by-cell basis. Although the current resolution of inversion detection by chromatid painting is on average $\sim 1$ MB based on spacing of the probe sets, and represents an order of magnitude improvement over traditional banding, this is by no means the limit of resolution that can be achieved. We demonstrate that detection of much smaller inversions is entirely possible with more densely targeted probe coverage, placing chromatid painting among approaches normally considered the purview of high-resolution cytogenetic methods such as interphase FISH (Mancini et al. 2000) or Fiber FISH (Korbel et al. 2007). However, we also note that resolution in terms of inversion detection should perhaps more precisely be viewed as "probability of detection," a more accurate way of viewing the fact that based on spacing of probe sets, smaller and smaller inversions can indeed be detected, but with smaller and smaller probability depending on the location of the breakpoints. Importantly, chromatid painting revealed that the spontaneous frequencies of inversions in both normal human fibroblasts and lymphocytes were quite low $(<0.3 \%)$, a favorable and critical factor when evaluating dose responses for inversions induced by various genotoxic agents including IR, where medical, environmental, and occupational exposures represent significant health concerns.

The realization of strand-specific hybridization has, at least to date, provided corroborating support for the assembly of the human genome database in terms of it being aligned and oriented correctly, as well as initial evidence that individuals themselves share similar sequence directionality. Analogous experiments conducted during the design of human chromosome 10 strand-specific probes revealed similar orientation of contigs along this chromosome as well (not shown). Interestingly, we also noted that dependent on probe set spacing, fluorescent signals did not always present as uniform visibly contiguous stretches. A possible explanation for this may be related to the variable state of chromatin condensation that chromosomes present throughout metaphase, prompting our speculation that strand-specific probe sets designed at specific and varying intervals may be useful for investigating chromosome compaction.

We provide evidence of the utility of chromatid painting strategies for comparative and evolutionary studies, demonstrating their likely potential to complement studies comparing human and distantly related species, as for example was recently reported with the Y chromosome (Hughes et al. 2012). In fact, directional genomic hybridization methodology could be used to develop probe sets to any species with suitable chromosomes whose genome has been sequenced, e.g., equine, canine, feline, and exotic or endangered species. Additionally, unlike conventional chromosome painting, chromatid painting facilitates simultaneous detection of both intrachromosomal (inversions) and inter-chromosomal rearrangements (dicentrics, translocations). And like chromosome painting, it is also amenable to multicolor combinatorial schemes of chromosome identification (Speicher et al. 1996). Lastly, the ability to use sequence- and strand-specific probe sets for standard FISH applications greatly expands their use for diagnostic and prognostic purposes.

Although inversions have been detectable at the resolution of traditional cytogenetics $(>10 \mathrm{Mb})$ for many years, there has been a paucity of methods for high-resolution global, unbiased inversion discovery. Therefore, it is largely unknown how common inversions really are, what their size distribution is, and to what extent they are associated with human disorders. 
Improved detection of inversions, especially small ones, such as that provided by directional genomic hybridization strategies, stands to facilitate efforts to characterize the contribution of inversions to structural variation in the human genome, thereby aiding investigation of underlying disease mechanisms. Ultimately, novel gene discovery and high-resolution inversion detection have the potential to benefit the diagnosis and treatment of a variety of disease states and disorders including cancer, autism, developmental delay, and idiopathic infertility.

Open Access This article is distributed under the terms of the Creative Commons Attribution License which permits any use, distribution, and reproduction in any medium, provided the original author(s) and the source are credited.

\section{References}

Antonacci F, Kidd JM, Marques-Bonet T, Ventura M, Siswara P, Jiang Z, Eichler EE (2009) Characterization of six human disease-associated inversion polymorphisms. Hum Mol Genet 18(14):2555-2566

Asou H, Eguchi M, Suzukawa K, Morishita K, Tanaka K, Date M, Hamamoto K, Kamada N (1996) Establishment of a myeloid leukaemia cell line (Kasumi-4) with $\mathrm{t}(9 ; 22 ; 11)(\mathrm{q} 34 ; \mathrm{q} 11 ; \mathrm{q} 13)$, $\operatorname{inv}(3)(\mathrm{q} 21 \mathrm{q} 26)$ and the EVI1 gene activation from a patient with chronic myelogenous leukaemia in blast crisis. Br J Haematol 93(1):68-74

Bailey SM, Brenneman MA, Goodwin EH (2004a) Frequent recombination in telomeric DNA may extend the proliferative life of telomerase-negative cells. Nucleic Acids Res 32(12):3743-3751

Bailey SM, Cornforth MN, Kurimasa A, Chen DJ, Goodwin EH (2001) Strand-specific postreplicative processing of mammalian telomeres. Science 293(5539):2462-2465

Bailey SM, Cornforth MN, Ullrich RL, Goodwin EH (2004b) Dysfunctional mammalian telomeres join with DNA double-strand breaks. DNA Repair (Amst) 3(4):349-357

Bailey SM, Goodwin EH, Cornforth MN (2004c) Strandspecific fluorescence in situ hybridization: the CO-FISH family. Cytogenet Genome Res 107(1-2):14-17

Bailey SM, Williams ES, Cornforth MN, Goodwin EH (2010) Chromosome orientation fluorescence in situ hybridization or strand-specific FISH. Methods Mol Biol 659:173-183

Baker M (2012) Structural variation: the genome's hidden architecture. Nat Methods 9(2):133-137

Bickmore W, Craig J (1977) Chromosome bands: patterns in the genome. Chapman and Hall, New York

Boyle S, Rodesch MJ, Halvensleben HA, Jeddeloh JA, Bickmore WA (2011) Fluorescence in situ hybridization with high-complexity repeat-free oligonucleotide probes generated by massively parallel synthesis. Chromosome Res 19(7):901-909
Cancer Genome Atlas N (2012) Comprehensive molecular characterization of human colon and rectal cancer. Nature 487(7407):330-337

Caudill CM, Zhu Z, Ciampi R, Stringer JR, Nikiforov YE (2005) Dose-dependent generation of RET/PTC in human thyroid cells after in vitro exposure to gamma-radiation: a model of carcinogenic chromosomal rearrangement induced by ionizing radiation. J Clin Endocrinol Metab 90(4):2364-2369

Chen JM, Cooper DN, Ferec C, Kehrer-Sawatzki H, Patrinos GP (2010) Genomic rearrangements in inherited disease and cancer. Semin Cancer Biol 20(4):222-233

Cornforth M (1998) Radiation-induced damage and the formation of chromosomal aberrations. In: Nickoloff JA, Hoekstra MF (eds) DNA damage and repair. Humana Press Inc, Totowa, pp 559-585

Cornforth MN, Eberle RL (2001) Termini of human chromosomes display elevated rates of mitotic recombination. Mutagenesis 16(1):85-89

Falconer E, Hills M, Naumann U, Poon SS, Chavez EA, Sanders AD, Zhao Y, Hirst M, Lansdorp PM (2012) DNA template strand sequencing of single-cells maps genomic rearrangements at high resolution. Nat Methods 9(11):1107

Feuk L (2010) Inversion variants in the human genome: role in disease and genome architecture. Genome Med 2(2):11

Goodwin E, Meyne J (1993) Strand-specific FISH reveals orientation of chromosome 18 alphoid DNA. Cytogenet Cell Genet 63(2):126-127

Goodwin EH, Meyne J, Bailey SM, Quigley D (1996) On the origin of lateral asymmetry. Chromosoma 104(5):345-347

Gribble SM, Prigmore E, Burford DC, Porter KM, Ng BL, Douglas EJ, Fiegler H, Carr P, Kalaitzopoulos D, Clegg S, Sandstrom R, Temple IK, Youings SA, Thomas NS, Dennis NR, Jacobs PA, Crolla JA, Carter NP (2005) The complex nature of constitutional de novo apparently balanced translocations in patients presenting with abnormal phenotypes. J Med Genet 42(1):8-16

Gruber Tanja A, Larson Gedman A, Zhang J, Koss Cary S, Marada S, Ta Huy Q, Chen S-C, Su X, Ogden Stacey K, Dang J, Wu G, Gupta V, Andersson Anna K, Pounds S, Shi L, Easton J, Barbato Michael I, Mulder Heather L, Manne J, Wang J, Rusch M, Ranade S, Ganti R, Parker M, Ma J, Radtke I, Ding L, Cazzaniga G, Biondi A, Kornblau Steven M, Ravandi F, Kantarjian H, Nimer Stephen D, Döhner K, Döhner H, Ley Timothy J, Ballerini P, Shurtleff S, Tomizawa D, Adachi S, Hayashi Y, Tawa A, Shih L-Y, Liang D-C, Rubnitz Jeffrey E, Pui C-H, Mardis Elaine R, Wilson Richard K, Downing James R (2012) An Inv(16)(p13.3q24.3)-encoded CBFA2T3-GLIS2 fusion protein defines an aggressive subtype of pediatric acute megakaryoblastic leukemia. Cancer Cell 22(5):683-697

Haluska FG, Finver S, Tsujimoto Y, Croce CM (1986) The t $(8$; 14) chromosomal translocation occurring in B-cell malignancies results from mistakes in V-D-J joining. Nature 324(6093):158-161

Hughes JF, Skaletsky H, Brown LG, Pyntikova T, Graves T, Fulton RS, Dugan S, Ding Y, Buhay CJ, Kremitzki C, Wang Q, Shen H, Holder M, Villasana D, Nazareth LV, Cree A, Courtney L, Veizer J, Kotkiewicz H, Cho TJ, Koutseva N, Rozen S, Muzny DM, Warren WC, Gibbs RA, Wilson RK, Page DC (2012) 
Strict evolutionary conservation followed rapid gene loss on human and rhesus Y chromosomes. Nature 483(7387):82-86

Imielinske M (2012) Mapping the hallmarks of lung adenocarcinoma with massively parallel sequencing. Cell 150:1107-1120

Korbel JO, Urban AE, Affourtit JP, Godwin B, Grubert F, Simons JF, Kim PM, Palejev D, Carriero NJ, Du L, Taillon BE, Chen Z, Tanzer A, Saunders ACE, Chi J, Yang F, Carter NP, Hurles ME, Weissman SM, Harkins TT, Gerstein MB, Egholm M, Snyder M (2007) Paired-end mapping reveals extensive structural variation in the human genome. Science 318(5849):420-426

Mancini M, Cedrone M, Diverio D, Emanuel B, Stul M, Vranckx H, Brama M, De Cuia MR, Nanni M, Fazi F, Mecucci C, Alimena G, Hagemeijer A (2000) Use of dual-color interphase FISH for the detection of inv(16) in acute myeloid leukemia at diagnosis, relapse and during follow-up: a study of 23 patients. Leukemia 14(3):364-368

Manning M, Hudgins L (2010) Array-based technology and recommendations for utilization in medical genetics practice for detection of chromosomal abnormalities. Genet Med 12(11):742-745

Mitelman F (2000) Recurrent chromosome aberrations in cancer. Mutat Res 462(2-3):247-253

Muhlmann-Diaz MC, Bedford JS (1995) Comparison of gamma-ray-induced chromosome ring and inversion frequencies. Radiat Res 143(2):175-180

Nowell P, Hungerford D (1960) A minute chromosome in chronic granulocytic leukemia. Science 132(3438)
Rowley JD (1973) Letter: a new consistent chromosomal abnormality in chronic myelogenous leukaemia identified by quinacrine fluorescence and Giemsa staining. Nature 243(5405): 290-293

Rowley JD (1990) Molecular cytogenetics: Rosetta stone for understanding cancer-twenty-ninth G. H. A. Clowes memorial award lecture. Cancer Res 50(13):3816-3825

Savage JR (1977a) Application of chromosome banding techniques to the study of primary chromosome structural changes. J Med Genet 14(5):362-370

Savage JRK (1977b) Assignment of aberration breakpoints in banded chromosomes. Nature 270:513-514

Speicher MR, Ballard SG, Ward DC (1996) Karyotyping human chromosomes by combinatorial multi-fluor FISH. Nat Genet 12(4):368-375

Tuzun E, Sharp AJ, Bailey JA, Kaul R, Morrison VA, Pertz LM, Haugen E, Hayden H, Albertson D, Pinkel D, Olson MV, Eichler EE (2005) Fine-scale structural variation of the human genome. Nat Genet 37(7):727-732

Yamada NA, Rector LS, Tsang P, Carr E, Scheffer A, Sederberg MC, Aston ME, Ach RA, Tsalenko A, Sampas N, Peter B, Bruhn L, Brothman AR (2011) Visualization of fine-scale genomic structure by oligonucleotide-based high-resolution FISH. Cytogenet Genome Res 132(4):248-254

Zou Y, Gryaznov SM, Shay JW, Wright WE, Cornforth MN (2004) Asynchronous replication timing of telomeres at opposite arms of mammalian chromosomes. Proc Natl Acad Sci U S A 101(35):12928-12933 